\title{
Star Formation History of the Hubble Ultra Deep Field: Comparison with the HDFN
}

\author{
Rodger I. Thompson \\ Steward Observatory, University of Arizona, Tucson, AZ 85721 \\ rthompson@as . arizona.edu \\ Daniel Eisenstein \\ Steward Observatory, University of Arizona, Tucson, AZ 85721 \\ deisenstein@as . arizona.edu \\ Xiaohui Fan \\ Steward Observatory, University of Arizona, Tucson, AZ 85721 \\ fan@as.arizona.edu \\ Mark Dickinson \\ National Optical Astronomy Observatories, Tucson, AZ 85719 \\ med@noao.edu \\ Garth Illingworth \\ University of California Santa Cruz, Santa Cruz, CA 95064 \\ and \\ Robert C. Kennicutt \\ Institute of Astronomy, University of Cambridge, Cambridge CB3 OHA UK and Steward \\ Observatory, University of Arizona, Tucson, AZ 85721 \\ robk@ast.cam.ac.uk
}

ABSTRACT 
We use the NICMOS Treasury and ACS HUDF images to measure the extinction corrected star formation history for 4681 galaxies in the region common to both images utilizing the star formation rate distribution function and other techniques similar to those employed with the NICMOS and WFPC2 images in the HDFN. Unlike the HDFN the NICMOS region of the HUDF appears to lack highly luminous and high star formation rate galaxies at redshifts beyond 3 . The HUDF provides a region that is completely uncorrelated to the HDFN and therefore provides and independent measure of the star formation history of the universe. The combined HUDF and HDFN star formation rates show an average star formation rate of $0.2 \mathrm{M}_{\odot} \mathrm{yr}^{-1}$. The average SFR of the combined fields at $\mathrm{z}=1-3$ is $0.29 \mathrm{M}_{\odot} \mathrm{yr}^{-1}$ while the average at $\mathrm{z}=4-6$ is $1.2 \mathrm{M}_{\odot} \mathrm{yr}^{-1}$. The SFRs at all redshifts are within $3 \sigma$ of the average over all redshifts.

Subject headings: galaxies: evolution, formation - stars: formation-cosmology: early universe

\section{Introduction}

The Hubble Deep and Ultra Deep Fields (Williams et al. (1996), Thompson et al. (1999),Dickinson (2000), Beckwith et al. (2006), Thompson et al. (2005)) are our deepest look at individual objects in the early universe. There are numerous investigations of the properties of galaxies at high redshift utilizing the observations in the Hubble Deep Field North (HDFN) (Lanzetta, Yahil \& Fernandez-Soto (1996), Madau, et al. (1996), Fernandez-

Soto, Lanzetta \& Yahil (1999), Thompson et al. (2001), Lanzetta et al. (2002), Thompson (2003) and others). The small size of the field, however, makes large scale structure the dominant error in projecting the properties of these galaxies such as the star formation history in the HDFN to the universe as a whole. The Hubble Ultra Deep Field (HUDF), with overlapping optical and near infrared images, provides an independent measure of the star formation history in a region completely uncorrelated to the HDFN. As such it provides an independent very deep field data point for determining the history of star formation in the universe. Unfortunately, even with a second independent deep field, large scale structure is still a major error source in the quest for the history of the evolution of baryonic matter into stars and galaxies. These new observations do confirm, however, that the star formation rate (SFR) at redshifts $\geq 1$ is significantly higher than the present day rate determined by Lilly et al. (1996). The results, however, are inconsistent with the star formation rates that continue to increase to redshifts of 6 and beyond as found by Lanzetta et al. (2002) in the HDFN. Within the errors it is consistent with high redshift SFRs found in other areas by 
Steidel et al. (1999), Barger, Cowie \& Richards (2000) and Yan et al. (1999). It is also consistent with the SFRs found in the Northern and Southern GOODs field by Giavalisco et al. (2004) which includes the HUDF.

The source extraction used in this investigation is relatively conservative and is based on the combination of ACS and NICMOS fluxes. For this reason we do not address the issues of reionization, population III stars, and the luminosity function at redshifts beyond 6. These have been investigated with these same images by Stanway, Bunker \& McMahon (2003), Stiavelli, Fall, \& Panagia (2004), Yan \& Windhorst (2004) and Bouwens et al. (2004) with conflicting conclusions on the number, nature and evolution of high redshift sources. Bouwnes et al. (2005) has attempted to reconcile some of these conclusions.

\section{Observations}

The observations are all taken in the HUDF centered at R.A. 3.32. 39.0. and Dec. $-27^{\circ} 47^{\prime} 29.1^{\prime \prime}$ (J2000) with the Advanced Camera for Surveys (ACS) and the Near Infrared Camera and Multi-Object Spectrometer (NICMOS). The ACS images were taken in the F450W, F606W, F775W and F850LP filters and the NICMOS images were taken in the F110W and F160W filters. The response curves for these filters is shown in Figure 1. The NICMOS images and data reduction are described in detail in Thompson et al. (2005) and the ACS images in Beckwith et al. (2006). The data used in this analysis are only from the $144^{\prime \prime}$ by $144^{\prime \prime}$ region where the NICMOS and ACS data overlap. In the rest of this manuscript we will refer to the overlap area as the NUDF. The extent of the region was determined by excluding pixels that had less than half the average integration time of pixels in the interior of the region. This occurred due to the dithering of center points of the images on the $3 \mathrm{x} 3$ tile pattern. Due to the significantly longer integration time per pixel, the ACS images have a higher signal to noise than the NICMOS images. $30 \%$ of the galaxies have F160W AB magnitudes of 28.0 or brighter which is the approximate completness limit of the NICMOS images The depth of the NICMOS images are roughly equivalent to the depth in the HDFN (Thompson 2003) while the ACS images are significantly deeper than the WFPC II images in the HDFN. This should be remembered in the comparison of the results between the two fields.

The use of the ACS images in this study is not exactly equivalent to the use of the WFPC2 images in the HDFN. The ACS images lack the very blue F300W band produced by WFPC2 in the HDFN. This makes the accuracy of low redshift determinations less than is achievable with the F300W band. For very low redshift $(z \lesssim 1)$ the F300W band provided a longer lever arm by sampling the SED at shorter wavelengths and for somewhat higher 
redshifts $(2 \lesssim z \lesssim 3.5)$ it monitored the Lyman break at lower redshifts than the ACS F450W filter. The ACS F850LP band also extends further to the red than the F814W band and significantly overlaps the the NICMOS F110W band. The deep ACS images in the F850LP provide high sensitivity to objects that would be dropouts in the WFPC2 F814W filter but detected in the ACS F850LP filter. The ACS images are better sampled than the WFPC2 images, although our rebinning of the ACS image to match the NICMOS resolution negates most of that advantage. The spatial resolution of the NICMOS images is slightly less than was available for the HDFN observations since the HST secondary was not refocused to achieve the best NICMOS resolution as was done in the two HDFN NICMOS campaigns. The difference in resolution is only about $10 \%$, however, and should not affect the photometry of extended sources.

\section{Data Reduction}

The data reduction of the NICMOS bands is described in detail in Thompson et al. (2005) except for the source extraction procedure which is described in $\S 3.1$. The ACS images were taken directly from the HST Treasury Archive maintained by MAST. The 0.03" ACS pixels were binned to match the $0.09^{\prime \prime}$ drizzled NICMOS images by simple addition of the ACS images in 3 by 3 pixel bins. The NICMOS images were then aligned to the ACS images by a three step process. The initial alignment was done by using World Coordinate System values in the headers of the images. The image alignment was then improved by cross correlating the F110W NICMOS image with the F850LP image in 0.1 pixel steps. The final alignment was done by finding the centroids of compact objects and aligning the images by the centroids. In all cases the NICMOS images were determined relative to the ACS images. The image positions of each NICMOS image relative to the ACS image were then used in the drizzle procedure to produce a mosaic NICMOS image that is accurately aligned with the ACS F850LP image and hence with the ACS images in all of its bands. In

each orbit the F160W image immediately followed the F110W image without moving the spacecraft, therefore, the alignments calculated for the F110W images were also applied to the F160W images. The 4 ACS and 2 NICMOS images used for the source extraction are available from MAST in the NICMOS UDF Treasury version 2 files. A mask was used to limit the extraction to only the area that contained valid NICMOS images, an area of $144^{\prime \prime}$ on a side which is less than half of the area of the ACS UDF field. 


\subsection{Source Extraction}

Unlike the creation of the NUDF Treasury catalog, source extraction is done in two steps and is based on the fluxes in all ACS and NICMOS bands, not just the two NICMOS bands. The first step is to identify pixels that have sufficient signal to noise to be considered source pixels and second to identify sources with the extraction program SExtractor, hereinafter SE (Bertin \& Arnouts 1996). Source pixel identification uses the procedure developed by Szalay, Connolly \& Szokoly (1999) for multiple band images. The first step in the process is the production of images with unit variances. For the NICMOS images we first constructed inverse quantum efficiency (QE) maps from the flat field images for the F110W and F160W camera 3 filters. The flat field images were drizzled in exactly the same way as the F110W and F160W images. Since the NICMOS flat fields are multiplicative this procedure produced inverse QE maps with the same weighting as the images. The F110W and F160W standard deviations from Figure 2 were multiplied by 1.8 to account for drizzle correlation (Fruchter and Hook 2002). The inverse QE maps were then multiplied by the standard deviations to produce a standard deviation map. The images were then divided by the standard deviation map to produce an image with unit standard deviation and hence unit variance. The same procedure was carried out with the ACS images except that only the drizzle weights were used rather than drizzled flat fields due to the much more uniform QE of the ACS detectors.

Each pixel is assigned a $g_{i}$ value of

$$
g_{i}=\frac{f_{i}-s_{i}}{\sigma_{i}}
$$

where $f_{i}$ is the flux, $s_{i}$ is the sky background, and $\sigma_{i}$ is the standard deviation and $i$ refers to the six bands. In this case the sky background has been subtracted and the fluxes come from the images that have been scaled to have unit variance which makes $\sigma$ values also unity. This makes the $g_{i}$ values equal to the $f_{i}$ values. Next each pixel is given a $y$ value which is

$$
y=\sum_{i=1}^{6} g_{i}^{2}
$$

for every pixel. The image formed by the $\mathrm{y}$ values is called the $\mathrm{R}$ map which should be a map of the $\chi^{2}$ values with 6 degrees of freedom. The histogram of the $\mathrm{R}$ values is shown in Figure 3 along with the probability distribution for $\chi^{2}$ for 4 degrees of freedom which is a much better fit than the distribution for 6 degrees of freedom. This is most likely because the variance of the F110W and F160W images has been overestimated and that the unit

variance images for these two bands are too low. The NICMOS images have a non-Gaussian 
distribution of noise values due to the correlation introduced by the drizzle procedure as described by Fruchter and Hook (2002). The tail is visible in Figure 3 of Thompson et al. (2005) on the negative side of the noise distribution. Flux from sources obscures the positive tail, however, it is present. Scaling of the NICMOS images to higher values reproduces the 6 degree of freedom distribution but the non-Gaussian tail produces spurious sources. For this reason we chose not to scale up the NICMOS images. This does not significantly affect the source extraction since the threshold $\mathrm{R}$ value is tuned to produce a thorough but conservative source extraction. The ACS images do not contain a non-Gaussian component since they were drizzled using a point target which does not spread flux between target pixels. In addition they have been rebinned in boxes of 3 by 3 pixels which greatly reduces any cross pixel correlation. This illustrates one of the difficulties in dealing with two data sets with significantly different signal to noise ratios and different noise characteristics. The boxes in Figure 3 show the observed histogram of $\mathrm{R}$ values represented by the asterisks minus the noise value of $\mathrm{R}$ as determined by the probability distribution traced by the solid line. The boxes represent the number of source pixels for a given $R$ value. The vertical dashed line at $\mathrm{R}=4.5$ is the $\mathrm{R}$ threshold used in this work for source pixels.

A new image is produced that has the $R$ value multiplied by 1000 for all pixels above the threshold and all other pixels at a random noise level with a standard deviation of $10^{-6}$. Without the minimal random noise the next step, source extraction with SE, aborts. Sources are extracted with SE in the two image method. The first image is the image derived from the $\mathrm{R}$ map and the second is the image in one of the ACS or NICMOS bands. Used in this way the sources are found from the first image but the photometry is extracted from the second. The configuration files for SE use an absolute flux threshold which is set so that all pixels identified by the Szalay et al. procedure are above the threshold and all pixels in the random noise field are orders of magnitude below the threshold. In this procedure the only role that SE plays in the source selection process is to enforce the criteria that any legitimate source must have a minimum area of 4 pixels and to identify the sources according to the deblending criteria. The value of DEBLEND_NTHRESH was set to 32 and the value of DEBLEND_MINCONT was set to 0.0001 . The extraction produced 4702 sources. Two of these sources are bright stars with clear diffraction spikes. 22 more unresolved objects were identified from the list of Pirzkal et al. (2005). These objects were not included in the determination of the star formation rates. Inspection of all of the sources revealed 19 sources that were suspect due to noise in the F110W band. Noise in the F110W band in generally due to cosmic ray persistence from hits that occurred before the beginning of an integration. As described in Thompson et al. (2005), the F110W band integration was always the first integration after earth occultation and subject to persistent hits that occurred during occultation. Hits that occur during the F110W integration or the immediately following F160W integration are 
removed by the cosmic ray hit removal software. The hits are recognizable by either linear features or cosmic ray spray geometries. The 19 suspect objects and the two stars were removed leaving 4681 sources. Removal of these sources had no affect on the conclusions of this paper. Although most of the remaining sources have no detectable signal in the two NICMOS bands the NICMOS upper bounds are useful in constraining the spectral energy distributions (SEDs).

The source extraction returns the flux in three apertures of diameters $0.54^{\prime \prime}, 1.08^{\prime \prime}$ and $3.06 "$ along with the isophotal and SE defined best and auto apertures. SE also returns a detection image with all of the pixels defined as part of an object labeled with the source ID. It is important to remember that in this case the pixels were not determined by SE but by the Szalay procedure described above. SE simply distributed the pixels into individual objects. In the following analysis we only use the aperture fluxes for determining the source properties and then sum over all pixels of the object to determine total fluxes.

Inspection of the images and extracted objects clearly indicates that there are valid sources that have been missed by the extraction process. For example none of the $\mathrm{z} \geq 7$ sources described by Bouwens et al. (2004) which only have flux in the NICMOS bands were extracted. Reduction of the detection threshold below 4.5 yields more sources but also starts to pick up sources that may be spurious. In this analysis we decide to take a conservative approach to source extraction and choose a threshold that has a high confidence level. A more aggressive source extraction, with a reliable check on source confidence may yield additional information on star formation at higher redshifts. The corrections for missed luminosity described in $\S 6.1$ will compensate in the SFR determination for objects missed due to our conservative threshold.

\section{Sensitivity and Aperture Correction}

The initial SED analysis produced a large number of sources with extremely blue NICMOS F110W to ACS F850LP colors but with reasonable NICMOS F160W to NICMOS F110W and ACS F850LP to ACS F775W colors. This led to a reanalysis of the NICMOS F110W and F160W sensitivities based on the large number of NICMOS Camera 3 observations of the solar analog star P330-E which has served as a NICMOS calibration star based on ground based photometry by Persson et al. (1998). This analysis revealed that the two bands were approximately $10 \%$ less sensitive than the analysis at the beginning of the NCS era had found which was based on relatively few observations. At the same time detailed aperture corrections were also performed which led to increased aperture corrections in going from the $0.54^{\prime \prime}$ diameter aperture measurements to the full power measurement for a point 
source. This new analysis is described more fully in Thompson (2005b). Due to the very high signal to noise ratio of the P330 observations the aperture corrections are far more accurate than the faint photometry and hence to not contribute to the noise budget of the observations. The net effect of these changes in calibration resulted in an increase in the NICMOS fluxes making the NICMOS to ACS colors consistent with the ACS to ACS colors and NICMOS to NICMOS colors. The fluxes in the Version 2.0 Treasury Catalogs in the MultiMission Archives at STScI (MAST) have not had the new sensitivities applied. Also note that the aperture fluxes and magnitudes in the catalog are for the photons detected in the stated apertures and must be corrected for the aperture function given in Table 1 or Thompson (2005b) when comparing with measurements with other instruments or facilities such as ACS. Table 1 lists the aperture corrections used in this work for the $0.54^{\prime \prime}$ diameter aperture used to define the galaxy SED. The ACS filter aperture corrections are taken from Sirianni et al. (2005).

Although the aperture corrections are for point sources, they are accurate for the small aperture $\left(0.54^{\prime \prime}\right)$ used for the parameter analysis. This small aperture flux is only used to determine the parameters of redshift, extinction and SED as described in $\S 5.1$. All photometric parameters are found by summing all of the pixels identified by SE as part of the object. They also have the merit of being accurately determined by this study and Sirianni et al. (2005). As a test an alternative method was carried out in which a scaled NICMOS PSF, as determined from the P330E image described above, was used to convolve the ACS images so that the PSF of the ACS image reasonably matched the NICMOS image. The NICMOS PSF was scaled since a convolution with the full width NICMOS PSF on the $3 \mathrm{x} 3$ binned ACS data would produce an ACS PSF that was broader than the NICMOS PSF. The full analysis from source extraction through SFR determination was performed and compared to the analysis using aperture corrections. In every redshift bin other than $\mathrm{z}=$ 6 the SFR was equal within $1 \sigma$. At $\mathrm{z}=6$ the SFRs were equal within $2 \sigma$ with the SFR from the convolved images being lower than the aperture correction SFR due to some galaxies having lower extinction and earlier SED values in the convolved image reduction compared to the aperture correction reduction. These changes were within the Poisson distribution of parameter errors as determined from the photometric error analysis described in $§ 6.2 .1$. We did not discover any systematic trends in redshift, SED or extinction between the two samples.

At this time there is some debate on whether NICMOS observations have a small nonlinear response in the sense that observations of faint sources may have lower sensitivity than brighter sources. To measure the sensitivity of our results to such an effect, the analysis was repeated using a NICMOS response function in both filters that was $10 \%$ less than the one determined above. The net change in SFR in each redshift bin was less than the errors 
shown in Figure 9. We therefore proceeded with the response functions determined from the observations of P330E.

\section{Data Analysis}

After the extraction of the source photometry the redshift, extinction, and SEDs of all of the sources are determined. The three parameters are determined using the $0.54^{\prime \prime}$ diameter aperture fluxes corrected by the factors in Table 1 . The $0.054^{\prime \prime}$ aperture fluxes are the most appropriate flux measurement for parameter determination for several reasons. Larger aperture or isophotal fluxes often are contaminated by flux from adjacent sources. Also the aperture fluxes include the highest signal to noise regions of the galaxy and experience with the HDFN has shown that they are the least susceptible to parameter perturbation due to photometric errors.

\subsection{Source Parameters}

As described in previous work (Thompson et al. (2001), Thompson (2003)), the source parameters of redshift, extinction and intrinsic SED are determined by a $\chi$ squared analysis of a comparison of the observed flux in the 6 photometric bands to the fluxes predicted by a suite of SEDs that have been numerically redshifted and extincted using the obscuration law of Calzetti et al. (2000). This analysis include both a determination of the extinction and an error term in the $\chi$ squared analysis that is proportional to the flux to account for errors in the absolute photometric calibration of ACS and NICMOS (see Eqn. 2 in Thompson (2003)). In this analysis the percentage error is set to $5 \%$ instead of $10 \%$. This better reflects the expected error in the ACS images and the recalibrated photometry match between NICMOS and ACS.

\subsubsection{Template SEDs}

Two changes were made from the previous analyses. A bluer and hotter calculated SED template was added to make 7 basic templates rather than the previous 6 templates. This was done because many of the galaxies were bluer than the bluest previous template which was for a 50 million year old galaxy. The new galaxy SED is a template calculated for a 10 million year old galaxy with a metallicity of 0.0001 and a Chabrier (2003) IMF from the SEDs of Bruzual \& Charlot (2003) based on the recommended Padova 1994 models. Also 
the previous 50 million year old template 6 model based on the BC96 models was replaced with a 50 million year old Bruzual \& Charlot (2003) model with a metallicity of 0.004 and a Chabrier (2003) IMF. These models have labels of m22 and m42 respectively in the Bruzual \& Charlot (2003) library. The first 5 templates based on observed SEDs are unchanged from the previous analysis as described in Thompson (2003). The SEDs are shown in Figure 4. Following past procedures the fluxes predicted by these base templates are interpolated between the base SEDs to produce 9 intermediate templates between each base template for a total of 61 total templates labeled $\mathrm{x} . \mathrm{y}$ where $\mathrm{x}$ is the base (1-7) and $\mathrm{y}$ is the increment (1-9). Note that the first 4 observed templates from Coleman, Wu \& Weedman (1980) were chosen by them to have very low extinction and template 5 from Calzetti, Kinney, \& Storchi-Bergmann (1994) is corrected to zero extinction. The small residual extinction in the observed galaxies has no significant effect on the derived SFRs, particularly because the vast majority of the star formation comes from galaxies with the latest 3 SEDs.

\subsubsection{Extinction}

For this analysis we have switched to the Calzetti obscuration law described by Calzetti et al. (2000). Otherwise the analysis is identical to the previous analysis. The E(B-V) values listed for the sources is the $\mathrm{E}(\mathrm{B}-\mathrm{V})_{s}$ value described in Calzetti et al. (2000) which is the value of $\mathrm{E}(\mathrm{B}-\mathrm{V})$ for the stellar continuum rather than the $\mathrm{E}(\mathrm{B}-\mathrm{V})$ for the nebular continuum.

\subsubsection{Redshift values}

The allowed redshift values ranged from 0 to 10, however, only the redshift range up to 6.5 was used in the star formation rate analysis. 14 of 4681 galaxies have redshifts above 6.5. Three galaxies have redshifts of $10.0, \mathrm{E}(\mathrm{B}-\mathrm{V})$ of 1.0 , the maximum, and SED template values of 2.0, the second earliest base template. It is clear that these galaxies had properties that were not well matched by the templates and were driven to the red boundaries of the extinction and redshift space. Visual inspection of the fluxes of these galaxies revealed that they are relatively faint galaxies with positive noise in the NICMOS F110W or F160W band

which was interpreted as a high redshift Lyman break. This is one of the problems that occurs when two data sets with quite different signal to noise properties are mixed. The problem was resolved by setting the F160W flux to zero for these objects, forcing the fit to depend more heavily on the optical bands. This is not the most satisfying solution but the contribution to the star formation rate of these objects is negligible. 
For 33 of the objects in the NUDF there are known redshifts from the VIMOS survey in the Chandra Deep Field South (CDFS). These are mostly at low redshift $(z \leq 1)$. For these 33 objects the extinction and SED were chosen by finding the minimum $\chi$ squared value among the calculated $\chi$ squared values in extinction and SED at the nearest grid redshift to the known redshift. This is different than what was done in Thompson (2003) where the redshift was set to the known redshift after the $\chi$ squared analysis which did not necessarily lead to the best SED and extinction for the known redshift. The histogram of redshift values is given in Figure 5.

An additional list of published and unpublished spectroscopic redshifts was prepared as part of the GOODS program. As a check on the validity of the photometric redshifts we compared the photometric and spectroscopic redshifts for the galaxies that had spectroscopic redshifts that were listed as equivalent to good or excellent in the heterogeneous rating methods of the lists. This resulted in 46 galaxies including, the VIMOS galaxies, and the comparison is shown in Figure 6. The references for the published redshifts are (Le Fèvre et al. (2004), Szokoly et al. (2004), Mignoli et al. (2005), Vanzella et al. (2005)). Only one galaxy redshift came from an unpublished observation. For the most part there is good correspondence between the spectroscopic and photometric redshifts with the exception of two catastrophic failures marked AGN and COMPOSITE in Figure 6. The AGN is the galaxy 2340 in our catalog and its optical image shows a clear diffraction spike from the nucleus. There is no AGN SED in our templates that can be used to get a good redshift. The second galaxy marked COMPOSITE in the figure is galaxy 2170 in our catalog. It is adjacent to galaxy $22181.5^{\prime \prime}$ away which has a photometric redshift of 0.5 equivalent to the spectroscopic redshift of 0.456. Although 2218 is 2 magnitudes fainter than 2170 it is possible that some spectral features contaminated the ground based spectrum of 2170 which had a slit width comparable to the separation of the galaxies. Currently there are not many spectroscopic redshifts in th NUDF for redshifts between 2.2 and 5.5. The reader is referred to Thompson (2003) for a spectroscopic versus photometric redshift comparison in the HDFN that has numerous examples in this region. The analysis method here is exactly the same except for the different ACS filters. The Lyman break enters the ACS F435W filter at a redshift of 2.1, therefore, for most of the range where we do not have spectroscopic confirmation the primary signature of photometric redshifts is well covered.

Figure 7 shows a plot of the F775W AB magnitude versus redshift. Superimposed on the figure are three lines showing the F775W magnitude of an $\mathrm{L}^{*}\left(3.4 \times 10^{10} \mathrm{~L}_{\odot}\right.$ bolometric luminosity) galaxy at the redshifts in the plot for the highest temperature SED (7) with no extinction, SED (7) with $\mathrm{E}(\mathrm{B}-\mathrm{V})=0.2$ and the earliest SED (1) with no extinction. It is clear that vast majority of sources lie within the boundaries of these lines. In the previous papers (eg. Thompson (2003)) the F160W magnitude was plotted instead of an optical 
magnitude. The much higher signal to noise of the optical data in this case makes it more instructive to use an optical magnitude. If we used the F160W band more than $2 / 3$ of the points would be null detections.

\subsection{Source 1810}

One source, $\mathrm{ID}=1810$, had photometric parameters of $\mathrm{z}=2.7, \mathrm{E}(\mathrm{B}-\mathrm{V})=1.0$ and $\mathrm{SED}$ $=6.7$, one of the hottest SEDs. With these parameters it had an SFR of 27,463 $\mathrm{M}_{\odot}$ per year, making the validity of these parameters very doubtful. The fit had gone to the extremes of the extinction which predicted a very large mid-infrared flux. If the extinction is set to zero the appropriate fit parameters are $\mathrm{z}=1.2$ and an $\mathrm{SED}=1.1$, a very early galaxy. Spitzer observations obtained in the GOODS program (Dickinson et al. 2006) showed IRAC fluxes consistent with the early galaxy at redshift 1.2 interpretation and inconsistent with strong thermal dust emission at a redshift of 2.7. A $3 \sigma$ upper limit of 18 microJanskys at 24 microns was provided by the MIPs observations. For these reasons the parameters for Source 1810 were set to their zero extinction values. With these parameters its contribution to the SFR at redshift 1 is negligible. No other source exhibited such an extreme SFR.

\section{The Nature of the Outliers}

The primary outlier in the redshift-magnitude diagram, Figure 7 is object 2340 adjusted to its spectroscopic redshift, the AGN galaxy discussed above. This is object KX10 in the table of $\mathrm{K}$ band selected xray sources in the CDFS (Croom, Warren \& Glazebrook 2001) and is identified as an AGN based on the MgII and [OII] spectral features. In fact the ACS images display faint diffraction spikes, presumably from the nucleus of the AGN.

There are several galaxies near redshift 3 that exceed the unextincted $\mathrm{L}^{*}$ magnitude but that is not unexpected. In fact the low number of greater than $\mathrm{L}^{*}$ galaxies is surprising when compared to the HDFN where there are 27 galaxies at redshifts between 4 and 6.5 with greater than $\mathrm{L}^{*}$ unextincted magnitudes. Galaxy 2888 is the only galaxy at $\mathrm{z}$ above 4 that has a greater than $\mathrm{L}^{*}$ unextincted magnitude. Its photometric redshift is 5.7 and a spectroscopic redshift of 5.83, a SED template number of 5.9 and an E(B-V) value of 0.02 . This galaxy was known before the position of the HUDF was picked and part of the decision on the location of the HUDF was the desire to include this galaxy. Detailed discussion of the scientific significance of the low number of high redshift greater than $\mathrm{L}^{*}$ galaxies is beyond the scope of this paper other than to comment that if highly luminous galaxies are associated with very over dense regions then they may be more strongly correlated than 
lower luminosity galaxies.

\subsection{Source Catalog}

A catalog of source properties is available in the electronic version of this paper. The catalog is far to large to be published in the print version. Each row has 69 data entries so it is not possible to print a stem of the catalog here. The catalog is a Right Ascension sorted catalog. Along with its internal id number each source has a cross reference to the Version 2 NICMOS UDF catalog available from MAST at the Space Telescope Science Institute and the ACS UDF Version 1 catalog available from the same source. A 0 in the cross-reference indicates that there was no match. Since the sources were selected from a combination of the ACS and NICMOS fluxes, most of the cross references to the Treasury catalog are 0. For the ACS matching sources, the ACS catalog position was required to be within 0.3 arc seconds of the NICMOS plus ACS position. In some cases a different morphology in the infrared images may produce a false no match indication. The $0.54^{\prime \prime}$ diameter aperture SFR is also provided. This is the SFR from the flux in the smallest photometric aperture. This is not the flux used in the calculation of the SFR in the previous section where the SFR for every detected pixel was used. The sum of the catalog SFRs will be substantially less than the SFR calculated in this paper. Users can adjust the rate by the ratio of the $0.54^{\prime \prime}$ diameter aperture fluxes to other fluxes of their choosing. The content and detailed format of the catalog is supplied in the electronic submission. All source numbers used in this paper refer to this catalog.

\section{Calculating the Star Formation Rates}

The procedure for calculating the SFRs is identical to the method used in previous publications (Thompson et al. (2001), Thompson (2003)) modulo the changes described above in the extinction law, the template SEDs and the details of the handling of the known redshift galaxies. For this reason only brief descriptions of the method are given in this publication and the reader is referred to the publications cited above for more detailed descriptions.

\subsection{The Star Formation Intensity Distribution}

The star formation distribution function $h(x)$, first developed by Lanzetta et al. (1999), and further discussed in Lanzetta et al. (2002) has proved to be an excellent measure of the 
total star formation rate at a given redshift range. The distribution has been discussed several times but a brief description is given here as the form of the distribution is not immediately intuitive. The star formation intensity $x$ is the SFR in solar masses per year per proper square kiloparsec. This is calculated for each pixel that is considered part of a galaxy. These are the pixels marked in the detection image returned by SE. For a given redshift interval the distribution function $h(x)$ is the sum of all the proper areas in an interval of $\mathrm{x}$, divided by that interval and divided by the comoving volume in cubic megaparsecs defined by the boundaries of the observed field and the redshift interval (Lanzetta et al. 1999). The relation between the SFR and $h(x)$ is given by equation 3

$$
S F R=\int_{0}^{\infty} x h(x) d x
$$

which shows that the star formation rate is first moment of $h(x)$. If the shape of $h(x)$ is invariant with redshift, the $h(x)$ as determined from low redshift observations can be used to correct the high redshift rates for the losses due to surface brightness dimming. This is done by sliding a smoothed version of the distribution as measured in the redshift 1 bin vertically until it matches the bright, large $x$ end of the distribution and then performing the integral in equation 3 over the adjusted $h(x)$. The smoothing is a 3 point smoothing for $\log h(x) \geq 10^{-4}$ which corresponds to $\log x \leq 1$ in the redshift 1 distribution. The height of the vertical adjustment was calculated by requiring the average of the smoothed $h(x)$ distribution at the 6 highest $\log x$ values for $\log x \leq 1$ to match the average of the of the observed $h(x)$ at the same $6 \log x$ values. If there are less than 6 valid values in the observed $h(x)$ then only the valid points are used. A similar method is used for the distribution without extinction correction but with the smoothing done over higher $\log h(x)$ values and lower $\log x$ values with only the first point used in determining the vertical scaling. The validity of the invariance with redshift and the empirical reasons why this might be so are discussed in detail in Thompson (2002). Briefly the change in the SFR intensity brought on by the expected smaller size in galaxies is offset by the expected lowering of the value of $\mathrm{M}_{*}$ in the Schecter distribution of masses. Also by using the extinction corrected SFR intensities the corrections are far less than matching to the SFR intensities that have not be corrected for extinction as was done in Lanzetta et al. (2002).

Figure 8 shows the measured SFR intensity distributions for redshifts 1 through 6 in unit redshift bins centered on the integer redshifts. The left hand column contains the distributions where all of the pixels have been corrected for the extinction found for the galaxy they reside in and the right hand column contains the distributions where no correction for extinction has been made. The smoothed $h(x)$ is represented by the solid line and observed values are represented by asterisks. It is easy to see the effects of surface brightness dimming 
at redshifts above 1 where the observed points dip below the smoothed line at low values of $\log x$. We will only use the results of the corrected distribution but show the uncorrected rates for comparison. Some of these comparisons are discussed in $\S 6.4$. The individual pixel areas are roughly 1 square kiloparsec at redshifts of 1 and greater. This is an optimal size as it represents roughly the smallest area over which the Schmidt law is valid (Kennicutt 1998), which is one of the empirical laws the invariance of $h(x)$ is based on (Thompson 2002). The corrections on the star formation rate for both extinction and surface brightness dimming by the $h(x)$ method are shown in Table 2. The larger surface brightness dimming correction for redshift 2 may be due to a slight mismatch in the $h(x)$ fitting which can be seen for $\log x$ values less than 0 in Figure 8. The correction is less than the error bars so there has been no attempt to adjust the value.

\subsection{The Star Formation Rates}

Figure 9 shows the star formation rates for the NICMOS region of the UDF binned in unit redshift bins centered on integer redshifts. The redshift range between 0.0 and 0.5 is not considered since small errors in redshift create very large errors in the calculated SFR for objects that close. The $1 \sigma$ error bars are the rms sum of photometric, number and large scale structure errors as described in $\S$ 6.2.1. The rates are calculated as described in $\S 6.1$ with the cosmological parameters $\mathrm{H}_{0}=70$., $\Omega_{0}=0.3$ and $\Lambda=0.7$. All SFRs from other works shown in the figure have been adjusted to this cosmology. The main features of the star formation history in the NUDF is a slightly elevated SFR at redshifts 1-3 and a slightly depressed rate at redshifts 4-6. The comparison with the star formation history in the HDFN is shown in Figure 10. Although the histories appear to be somewhat dissimilar they are within $1 \sigma$ of the HDFN error bars for all redshifts. The smaller error bars in the UDF are due to the increased number of sources and the higher signal to noise of the ACS photometry.

\subsubsection{Errors in the SFR}

There are several sources of errors in determining the SFR history of the NUDF. Some of these can be quantitatively measured while others, such as extinction not conforming to the extinction formula used in the analysis, are more difficult to measure. The first source of error addressed in photometric error. Photometric error is addressed by randomly altering the fluxes in each band for each source in a Gaussian distribution based on the measured $1 \sigma$

flux error in each band for the source and recomputing the SFR for each redshift bin. This 
is done 100 times using the $1 \sigma$ flux error computed by SE for each band. This procedure incorporates not only the flux errors in the computed 1500 Angstrom flux but also the changes in redshift, extinction and derived SED that flux errors can introduce. The SFR calculation is done in exactly the same manner as described in $\S 6.1$ which gives the error in SFR for a given redshift bin rather than on a galaxy by galaxy basis. The photometric error in each redshift bin is calculated from the standard deviation of the 100 different SFRs for each bin. The photometric error is then root mean square combined with the shot noise error taken as

$$
S F R_{\text {shotnoise }}=\frac{\sqrt{\sum s f r^{2}}}{\sum s f r}
$$

The other source of error is large scale structure as described in $\S 6.2 .2$. The total error, as shown in Figure 9 is the root mean square of the photometric and number errors with the large scale structure error. The contributions from each source of error for each redshift bin is tabulated in Table 3 .

\subsubsection{Calculation of Large Scale Structure Error}

The uncertainty in the star formation rate due to sample variance, also referred to as large-scale structure, can be estimated by computing the variance in the density field for the sample volume. The variance of the density field is given exactly by integrals of the two-point correlations; higher-order correlations and non-Gaussianity enter only into higher moments of the distribution of densities.

The survey volume in the case of the NUDF is very extended along the line-of-sight compared to the transverse size and hence to the wavelength of the fluctuations that dominate the variance. This makes Limber's equation a good approximation (Limber 1953) The angular power spectrum of a given selection of objects is given in flat cosmologies by

$$
P_{2}(K)=\frac{1}{K} \int d k P(k) f(K / k)=\int \frac{d r_{a}}{r_{a}^{2}} P\left(K / r_{a}\right) f\left(r_{a}\right)
$$

where $K$ is the angular wavenumber in $\mathrm{rad}^{-1}, r$ is a separation on the sky in $h^{-1} \mathrm{Mpc}, k$ is the spatial wavenumber in $h \mathrm{Mpc}^{-1}, P_{2}(K)$ is the angular power spectrum, and $P(k)$ is the non-linear spatial power spectrum; see Baugh \& Efstathiou (1994) for more discussion and notation. We have suppressed the redshift dependence of the power spectrum. Cosmology enters through the coordinate distance $r_{a}$ to a redshift $z$, and $r_{a}=K / k$. Finally, $f(r)$ is the square of the probability distribution of the coordinate distance to the selected objects; this 
is trivially related to the redshift distribution of the objects. For objects spread uniformly in $r_{a}$ between two limits $r_{1}$ and $r_{2}$, we simply have $f(r)=\left(r_{2}-r_{1}\right)^{-2}$ in that region and zero elsewhere.

For photometric-redshift selected samples at $z \gtrsim 1$, it is usually the case that one has selected a sample for which the coordinate distance does not vary much through the slab. In this case, one can treat $P$ and $r_{a}$ as constant in the region where $f$ is non-zero. This yields the form

$$
P_{2}(K)=\frac{P\left(K / r_{a}\right)}{r_{a}^{2}} \int d r_{a} f\left(r_{a}\right) \approx \frac{P\left(K / r_{a}\right)}{r_{a}^{2} \Delta r}
$$

where the last form assumes a uniform distribution of a thickness $\Delta_{r}=r_{2}-r_{1}$, which in turn is $c \Delta z / H(z)$. It should be noted that distributions with softer cutoffs actually yield smaller clustering amplitudes (but correlate neighboring slabs).

With the angular power spectrum of the selected objects in hand, one can compute the variance in a given angular survey region. This is easiest for a circular patch, for which the answer is simply

$$
\sigma^{2}=\frac{2}{\pi} \int K d K P_{2}(K)\left[\frac{J_{1}^{2}(K a)}{K a}\right]^{2} \approx \frac{2}{\pi \Delta r} \int k d k P(k)\left[\frac{J_{1}^{2}(k R)}{k R}\right]^{2}
$$

where $a$ is the angular radius of the patch and $R$ is the transverse radius of the patch (in length units, not angles). The second equality uses the uniform approximation given above. The difference between the variance of a square region and a circular one of the same size is small, negligible for this estimate. We therefore treat the NUDF as a circle of $81^{\prime \prime}$ radius.

Using the standard $\Lambda \mathrm{CDM}$ cosmology and a simple model for the non-linear power spectrum, we estimate that the rms variations in the density for patches the size of the NUDF and unit redshift thickness are about $0.3 \sigma_{8}$ for $z=1$ to $z=4$, growing to about $0.4 \sigma_{8}$ at $z=6$. Here, $\sigma_{8}$ is the rms fluctuations of the galaxies in spheres of $8 h^{-1} \mathrm{Mpc}$ comoving radius. Typically, one would estimate $\sigma_{8} \approx 1$ for most populations of star-forming galaxies, although this may be a slight overestimate, as recent clustering results at $z \gtrsim 1$ have been tending to come in slightly smaller. For example, the correlation lengths of order $3-4 h^{-1} \mathrm{Mpc}$ in Coil et al. (2004) and Le Fèvre et al. (2005) would correspond to $\sigma_{8} \approx 0.8$. However, the results of Adelberger et al. (2005) are $4-4.5 h^{-1} \mathrm{Mpc}$, and so we keep $\sigma_{8}=1$ to be conservative. In detail, different populations of galaxies have different clustering, and we are interested in the star-formation-weighted bias. The systematic errors in Limber's equation and our subsequent approximations are small compared to the uncertainties in the clustering amplitude.

Hence, we expect rms fractional uncertainties in the star formation rate of about $30 \%$ per unit redshift. This is large enough that one does not expect the distribution to be 
Gaussian-density fluctuations are typically skew-positive-but our computation from the power spectrum does get a correct measurement of the variance of the distribution. The results of the calculation for the area of the NUDF are given in Table 3 in the column labeled LSS err. The error bars in Figure 9 indicate the combined photometric and large scale structure errors.

\subsection{Galaxies that Contribute the Majority of the Star Formation}

Table 4 lists the number of galaxies that contribute $90 \%$ of the SFR in each redshift bin and the total number of galaxies in the redshift bin. In all cases less than half of the galaxies contribute $90 \%$ of the SFR. In most cases less than $25 \%$ of the galaxies contribute $90 \%$ of the SFR. It should be emphasized that the calculation of galaxy SFRs was done by integrating the SFR in all pixels defined by SE as part of a galaxy. The total SFR at each redshift was calculated by the method described in $\S 6.1$ which accounts for flux missed in the source extraction by SE.

Comparison of the number of galaxies contributing $90 \%$ of the SFR to the number of galaxies in Table 4 indicates that the majority of star formation appears to occur during periods of enhanced star formation in galaxies rather than constant sustained star formation over a long period of time. If the hierarchical model of galaxy formation is invoked this may be during the time when mergers occur and leads to the interesting speculation that perhaps the star formation history of galaxies is also a monitor of the hierarchical formation history. In that case the numbers in Table 4 indicate the percentage of galaxies with magnitudes brighter than the magnitude limit of the sample that are in the starburst/merger mode at that epoch.

There is clearly a deficit of high SFR galaxies at redshifts of 4 and 5 which is reflected in the lower total SRF at those redshifts. The highest SFR galaxies at redshifts of 4 and 5 have SFRs that are roughly 10 times less than the SFRs for galaxies at lower redshifts. The lack of high SFR regions is also demonstrated in the $h(x)$ plots in Figure 8. As pointed out earlier this is in contrast to the results found in the HDFN and may be the result of the effects of LSS. Again this may reflect the conclusion that the majority of total star formation occurs in the few galaxies with the highest SFR and that the lack of such galaxies in a region of space and redshift range results in a lower total star formation for that region. It may also be true that if the star formation rate reflects the merger rate then the high SFR galaxies may reside in denser regions of space and they may be more clustered than the total distribution of matter in the universe. 


\subsection{Discussion of Physical Insights from the $h(x)$ Distribution}

The star formation intensity distributions presented in Figure 8 present a different way of looking at the history of star formation than the galaxy by galaxy view. The proper areas of the pixels at redshifts of 1 and beyond are roughly 1 sq. kiloparsec which is the area where Schmidt's law relating SFR to gas column density becomes valid (Kennicutt 1998). The star formation intensity distribution shows the distribution of the star formation rate per unit area as opposed to star formation rate per galaxy. For example it shows that at redshifts of 4 and 5 there is a lack of intense star formation areas as well as a lack of high SFR galaxies. Invoking the Schmidt law this may mean that there is a lack of galaxies with areas of high gas density at these redshifts in the NUDF region. Somewhat hidden in the log-log plot over a large range of values is that the intensity distribution at $x$ values greater than $1 \mathrm{M}_{\odot}$ per year per proper sq. kiloparsec is dropping sharply (Thompson 2002) and that the probability of finding areas with SFRs of $100 \mathrm{M}_{\odot}$ per year per proper sq. kiloparsec is quite rare.

At fainter intensities it is clear that surface brightness dimming is removing galaxies and parts of galaxies from detection. For photon counting instruments such as ACS and NICMOS the surface brightness dimming goes as $(1+z)^{-4}$ which is reflected in the figure. Note however that in the star formation density distribution derived from the redshift 1 results in the HDFN that $60 \%$ of the total star formation occurs in the distribution greater than $\log (\mathrm{x})=-.25$ and $95 \%$ from the distribution greater than $\log (\mathrm{x})=-1.25$ (Thompson et al. 2001) therefore we are still seeing most of the star formation even at higher redshift. This adds to our earlier conclusions that most of the star formation occurs in high SFR galaxies by saying that most of the star formation occurs in high SFR areas.

\section{Combined Star Formation Rates From the HDFN and the NUDF}

One of the major goals of the NUDF observations was to measure the SFR history in a region that was completely uncorrelated with the HDFN. Figure 10 shows the SFR history of the HDFN as determined by Thompson (2003) along with the SFR history of the NICMOS UDF determined in this work. Although the areas of the two fields are roughly similar the UDF images, due to the ACS observations, are considerably deeper. To get the combined SFR history of the two fields we weighted the SFR in each field by the number of galaxies in each redshift bin. The error bars in the HDFN values are from Thompson (2003). The average SFR of the combined fields at $\mathrm{z}=1-3$ is $0.29 \mathrm{M}_{\odot} \mathrm{yr}^{-1}$ while the average at $\mathrm{z}=4-6$ is $1.2 \mathrm{M}_{\odot} \mathrm{yr}^{-1}$. The SFRs at all redshifts are within $3 \sigma$ of the average over all redshifts. Deep measurements over a much larger area are needed to accurately determine SFR history of 
the universe as opposed to just in the two small deep fields.

\section{Conclusions}

The NUDF provides an independent measure of the star formation history at high redshifts. Unlike the HDFN, the NUDF appears to have a paucity of very luminous and high SFR galaxies past a redshift of approximately 3. This lack is probably due to variances in population due to large scale structure. The variances between the fields highlights large scale structure as a major error in the determination of the SFR history of the universe. The combined data of the HUDF and the HDFN show a peak in the SFR at a redshift of 2 with a decline toward higher redshifts. Deep optical and infrared images over a very large area will be needed to quantify the SFR history of the universe. Progress on this quest can be made if the infrared channel of the WFC3 becomes operational on HST. True progress may require a dedicated mission similar to some of the missions developed as part of the Origins Probe mission studies commissioned by NASA.

We wish to than an anonymous referee for helpful comments that improved the paper. We wish to thank Eros Vanzella for providing unpublished redshifts that have been used for spectroscopic comparison to our photometric redshifts. This article is based on data

from observations with the NASA/ESA Hubble Space Telescope, obtained at the Space Telescope Science Institute, which is operated by the Association of Universities for Research in Astronomy under NASA contract NAS 5-26555. RIT, DE, XF and GI are funded in part by NASA Grant HST-GO-09803.01-A-G from the Space Telescope Science Institute.

\section{REFERENCES}

Adelberger, K.L., Steidel, C.C., Pettini, M., Shapley, A.E., Reddy, N.A., \& Erb, D.K., 2005, ApJ, 619, 697

Aussel, H. et al. 1999, A\&A, 342, 313

Barger, A.J., Cowie, L.L., Richards, E.A. 2000, AJ, 119, 2092

Baugh, C.M., \& Efstathiou, G. 1994, MNRAS, 267, 323

Beckwith, S. et al. 2005, in preparation

Bertin,E., \& Arnouts, S. 1996, A\&A, 117, 393 
Bouwens, R.J. et al. 2004, ApJ, 616, L79

Bouwens, R.J. et al. 2004, ApJ, in press, astro-ph/0509641 v4

Bruzual, G. \& Charlot, S., 1996

Bruzual, G. \& Charlot, S., 2003, MNRAS, 344, 1000

Calzetti, D., Kinney, A.L., \& Storchi-Bergmann, T. 1994, ApJ, 429, 582

Calzetti, D., et al. 2000, ApJ, 533, 682

Chabrier, G. 2003, PASP, 115, 763

Coil, A.L., et al., 2004, ApJ, 609, 525

Coleman, G. D., Wu, C. C., Weedman, D. W. 1980, ApJS, 43, 393

Croom, S.M., Warren, S.J. \& Glazebrook, K. 2001, MNRAS, 328, 1506

Dickinson, M. 2000, Phil. Trans. R. Soc. London A., 358, 2001

Dickinson, M. et al. 2006, in preparation

Fernandez-Soto, A., Lanzetta, K.M., \& Yahil, A. 1999, ApJ, 513, 34

Fruchter, A.S. \& Hook, R.N. 2002, PASP, 114, 144

Giavalisco, M. et al. 2004, ApJ, 600, L103

Kennicutt, K.C., Jr. 1998, ARA\&A, 36, 189

Lanzetta, K.M., Yahil, A., \& Fernandez-Soto, A. 1996, Nature, 381, 79

Lanzetta, K.M. et al. 1999, in ASP Conf. Ser. 191, Photometric Redshifts and High-Redshift Galaxies, ed. R.J. Weymann, L.J. Storrie-Lombardi, M. Sawicki, \& R.J. Brunner (San Francisco ASP), 223

Lanzetta, K.M. et al. 2002, ApJ, 570, 492

Le Fèvre, O., et al. 2004, A\&A, 428, 1043

Le Fèvre, O., et al., 2005, A\&A, 439, L877

Lilly, S.J. et al. 1996, ApJ, 460, L1

Limber, D.N. 1953, ApJ, 117, 134 
Madau, P. et al. 1996, MNRAS, 283, 1388

Madau, P., Pozzetti, L., \& Dickinson, M. 1998, ApJ, 498, 106

Mignoli, M. et al. 2005, A\&A, 437, 883

Persson, S.E. et al. 1998, AJ, 116, 2475

Pirzkal, N. et al. 2005, ApJ, 622, 319

Sirianni, M. et al. 2005, PASP, 117, 1049

Stanway, E.R., Bunker, A.J., McMahon, R.G. 2003, MNRAS, 342, 439

Steidel, C.C., Adelberger, K.L., Giavalisco, M., Dickinson,M., \& Pettini, M. 1999, ApJ, 519, 1

Stiavelli, M., Fall, S.M., \& Panagia, N. 2004, ApJ, 600, 508

Szalay, A.S., Connolly, A.J., \& Szokoly, G.P. 1999, AJ, 117, 68

Szokoly, G. P. et al. 2004, ApJS, 155, 271

Thompson et al. 1999, AJ, 117, 17

Thompson, R.I., Weymann, R.J. \& Storrie-Lombardi, L.J. 2001, ApJ, 546, 694

Thompson, R.I. 2002, ApJ, 581, L85

Thompson, R.I. 2003, ApJ, 596, 748

Thompson, R.I. et al. 2005, AJ, 130, 1

Thompson, R.I. 2005 in the Proceedings of the 2005 HST Calibration Workshop, A. Koekemoer, P. Goudfrooij, and L. Dressel, eds., Space Telescope Science Institute, in press.

Vanzella, E. et al. 2005, A\&A, 434, 53

Williams, R.E., et al. 1996, AJ, 112, 1335

Yan, L. et al. 1999, ApJ, 519, L47

Yan, H. \& Windhorst, R.A. 2004, ApJ, 612, L93 
Table 1. Aperture corrections for the 0.54" diameter aperture. As expected the aperture corrections increase starting with the F850LP band where a combination of scattering and the diffraction width limited PSF start to become significant relative to the aperture diameter. The NICMOS aperture corrections were derived from stellar observations as described in the text.

\begin{tabular}{ccccccc}
\hline \hline Filter & F445W & F606W & F775W & F850LP & F110W & F160W \\
\hline Correction Factor & 1.15 & 1.14 & 1.15 & 1.21 & 1.43 & 1.55 \\
\hline
\end{tabular}


Table 2. SFR corrections for extinction and surface brightness dimming. The volume in the SFR units is the comoving volume.

\begin{tabular}{ccccc}
\hline \hline & & & Extinction and & Missing \\
z & SFR $\left(\mathrm{M}_{\odot} y r^{-1} M p c^{-3}\right)$ & $\begin{array}{c}\text { Extinction Corrected } \\
\text { SFR }\left(\mathrm{M}_{\odot} y r^{-1} M p c^{-3}\right)\end{array}$ & $\begin{array}{c}\text { Dimming Corrected } \\
\text { SFR }\left(\mathrm{M}_{\odot} y r^{-1} M p c^{-3}\right)\end{array}$ & $\begin{array}{c}\text { Luminosity }{ }^{\mathrm{a}} \\
(\%)\end{array}$ \\
\hline 1 & 0.017 & 0.21 & 0.21 & 0 \\
2 & 0.021 & 0.30 & 0.40 & 24 \\
3 & 0.13 & 0.29 & 0.32 & 10 \\
4 & 0.027 & 0.082 & 0.094 & 13 \\
5 & 0.014 & 0.034 & 0.041 & 17 \\
6 & 0.019 & 0.10 & 0.13 & 17 \\
\hline
\end{tabular}

aThis is the percentage of missed luminosity between the extinction corrected SFR and the extinction corrected SFR which has also been corrected for surface brightness dimming. 
Table 3. Contributions to the total SFR error in $\mathrm{M}_{\odot} \mathrm{yr}^{-1} \mathrm{Mpc}^{-3}$. The column labeled Std. Dev. is the standard deviation of the SFRs computed in the 100 calculations of the SFR with random photometric errors added to the observed fluxes. The column marked SFR shot noise is the error computed in equation 4. The total errors are the root mean square sum of the individual errors. All errors are in units of $\mathrm{M}_{\odot} \mathrm{yr}^{-1} \mathrm{Mpc}^{-3}$ not fractions which accounts for the lower errors in regions of low SFR.

\begin{tabular}{cccccc}
\hline \hline Z & SFR & Std. Dev. & SFR shot noise & LSS err. & Total error. \\
\hline 1 & 0.208 & 0.036 & 0.056 & 0.073 & 0.099 \\
2 & 0.398 & 0.064 & 0.100 & 0.140 & 0.180 \\
3 & 0.322 & 0.057 & 0.110 & 0.110 & 0.160 \\
4 & 0.094 & 0.011 & 0.017 & 0.033 & 0.039 \\
5 & 0.041 & 0.006 & 0.006 & 0.014 & 0.016 \\
6 & 0.126 & 0.026 & 0.053 & 0.044 & 0.074 \\
\hline
\end{tabular}


Table 4. The table lists the number of galaxies that contribute $90 \%$ of the total SFR for a redshift bin and the total number of galaxies in the redshift bin. The SFR for the galaxy with the highest, third highest, and tenth highest SFR along with the SFR of the galaxy with the lowest SFR, but still in the group of galaxies that contribute $90 \%$ of the SFR, is also given.

\begin{tabular}{ccccccc}
\hline \hline & $\mathrm{z}=1$ & $\mathrm{z}=2$ & $\mathrm{z}=3$ & $\mathrm{z}=4$ & $\mathrm{z}=5$ & $\mathrm{z}=6$ \\
\hline Galaxies contributing 90\% of the SFR & 182 & 118 & 171 & 163 & 98 & 14 \\
Total number of galaxies & 1518 & 929 & 928 & 509 & 218 & 64 \\
Highest SFR in M $\odot \mathrm{yr}^{-1}$ & 425 & 899 & 1600 & 138 & 43 & 309 \\
Third Highest SFR & 260 & 582 & 347 & 81 & 20 & 59 \\
Tenth Highest SFR & 33 & 96 & 56 & 22 & 11 & 21 \\
Lowest SFR in the 90\% group & 1.3 & 4.1 & 2.9 & 1.1 & 0.8 & 12 \\
\hline
\end{tabular}




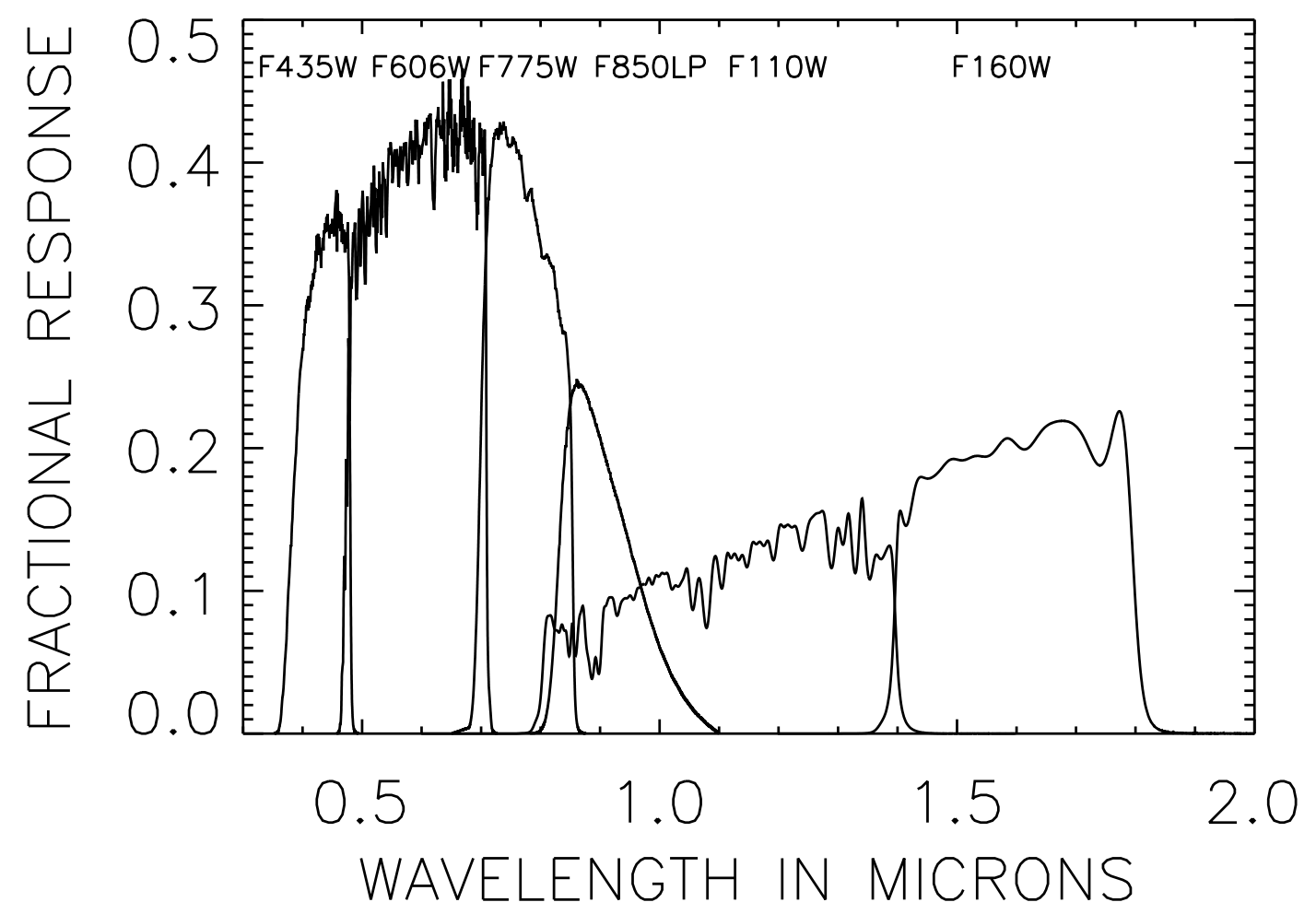

Fig. 1.- The response functions for the ACS and NICMOS filters used in the HUDF observations. The response functions are the filter transmission multiplied by the detector quantum efficiency at each wavelength. 

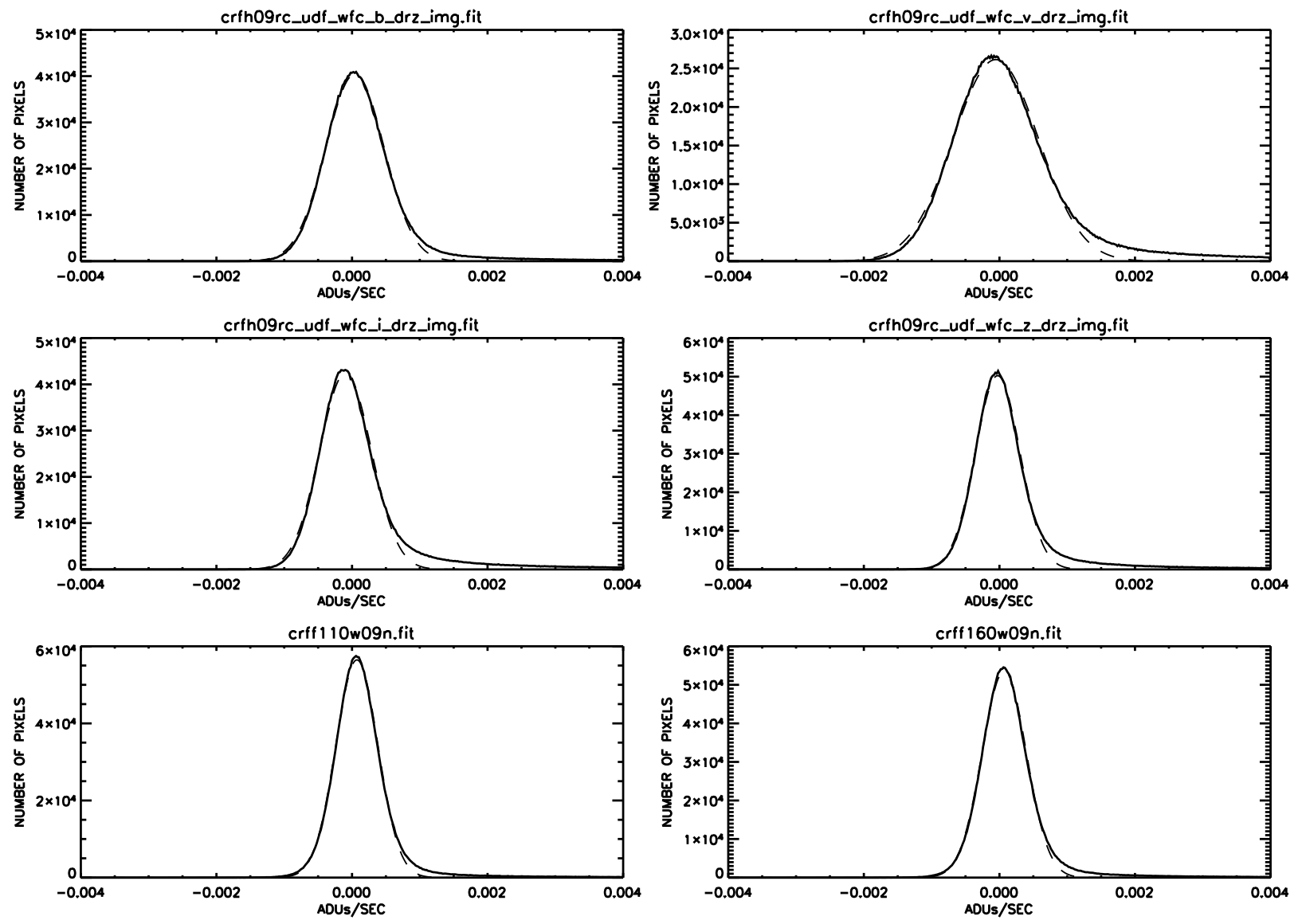

Fig. 2.- Histograms of the distribution of pixel values in the six images used in this study. The signals are in adus per second. The dashed line is a Gaussian fit to the distribution. The width of the Gaussian is used to find the average standard deviation of the noise in the image. The positive side power above the Gaussian fits is due to real sources in the image. 


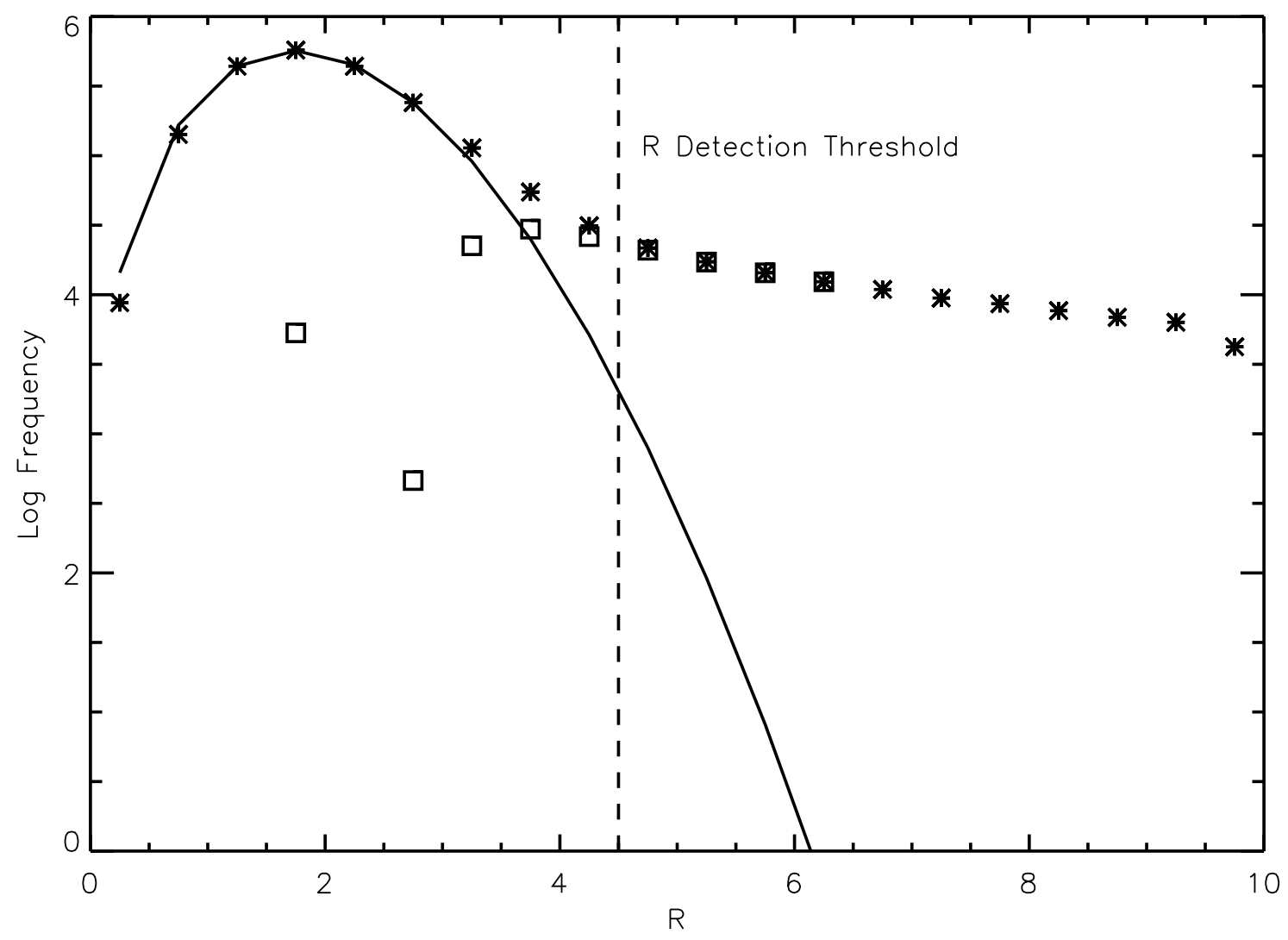

Fig. 3.- Histogram of the Szalay et al. $\mathrm{R}$ values as described in the text for the pixels in the NICMOS region of the UDF. The dashed line indicates the threshold $\mathrm{R}$ value of 4.5 for source pixels. The asterisks are the histogram values, the solid line is the $\mathrm{R}$ probability distribution for 4 degrees of freedom and the squares are the histogram values minus the 4 degree of freedom probability distribution. See $\S 3.1$ for a discussion of the method. 


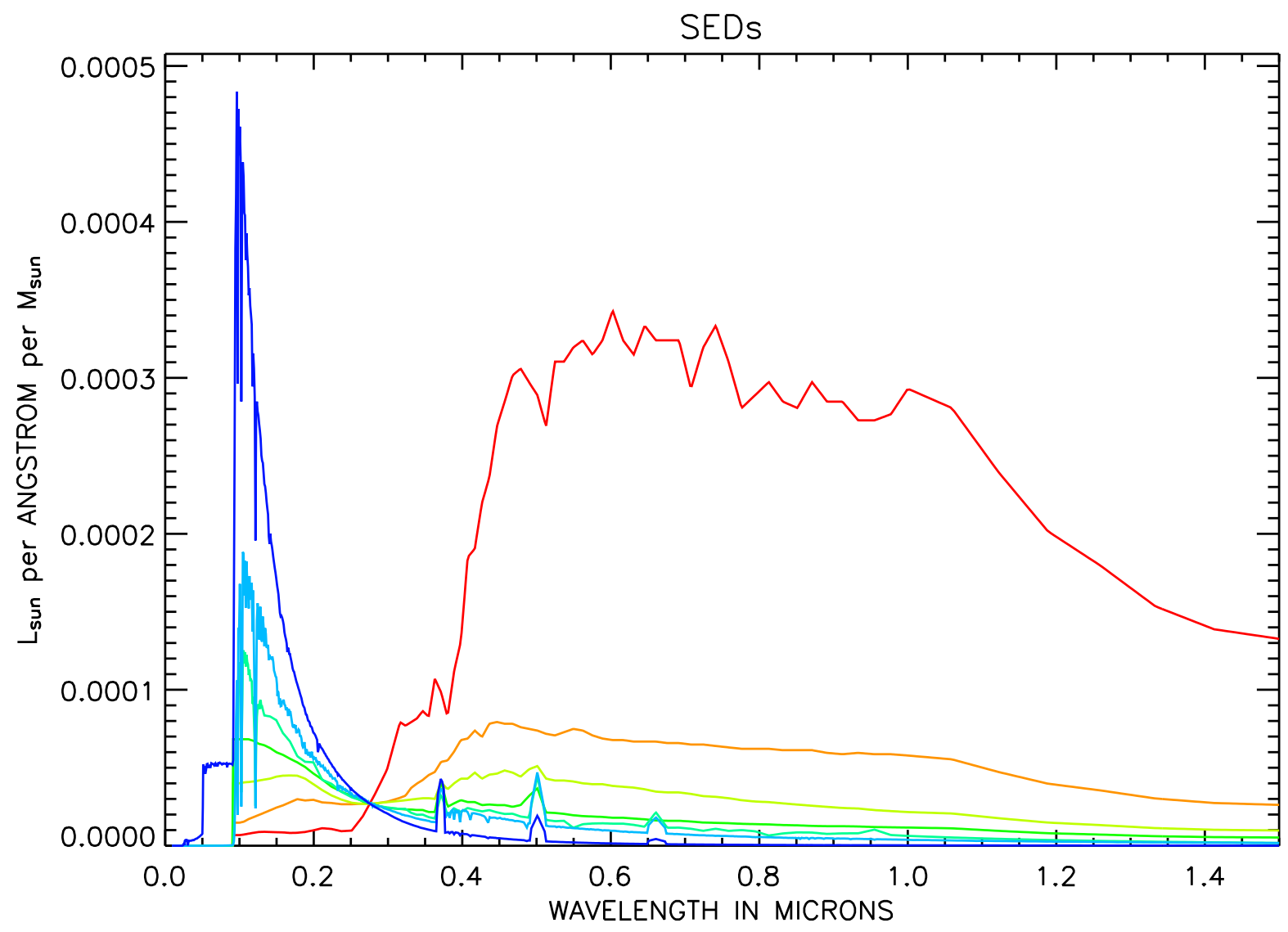

Fig. 4. - The 7 template SEDs used in this analysis. The two bluest templates have been calculated from the models of Bruzual \& Charlot (2003) as described in the text. 


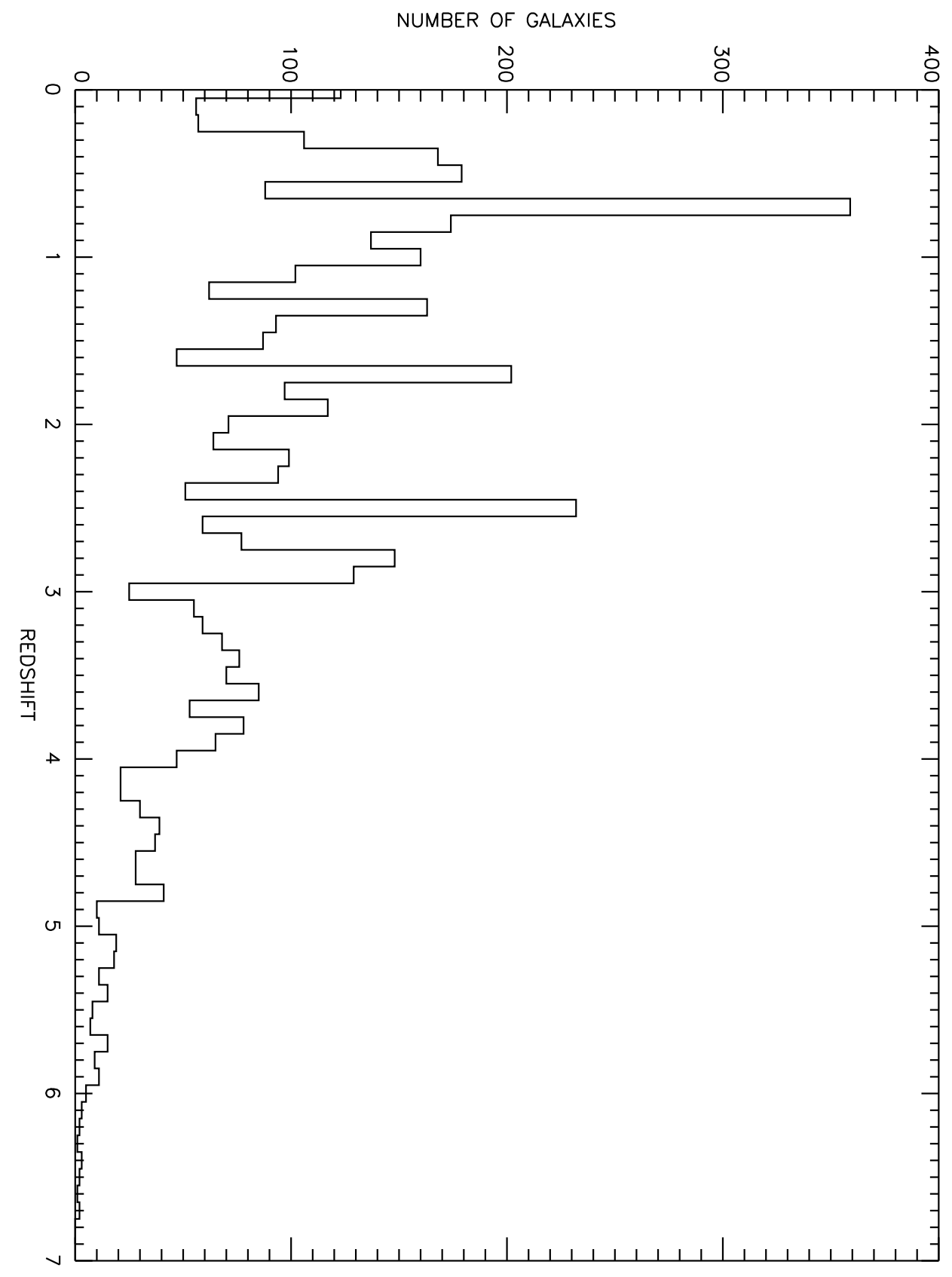

Fig. 5.- A histogram of the redshift values for the galaxies in the NICMOS region of the UDF. Only galaxies in the redshift region between 0.5 and 6.5 are used in this analysis. 


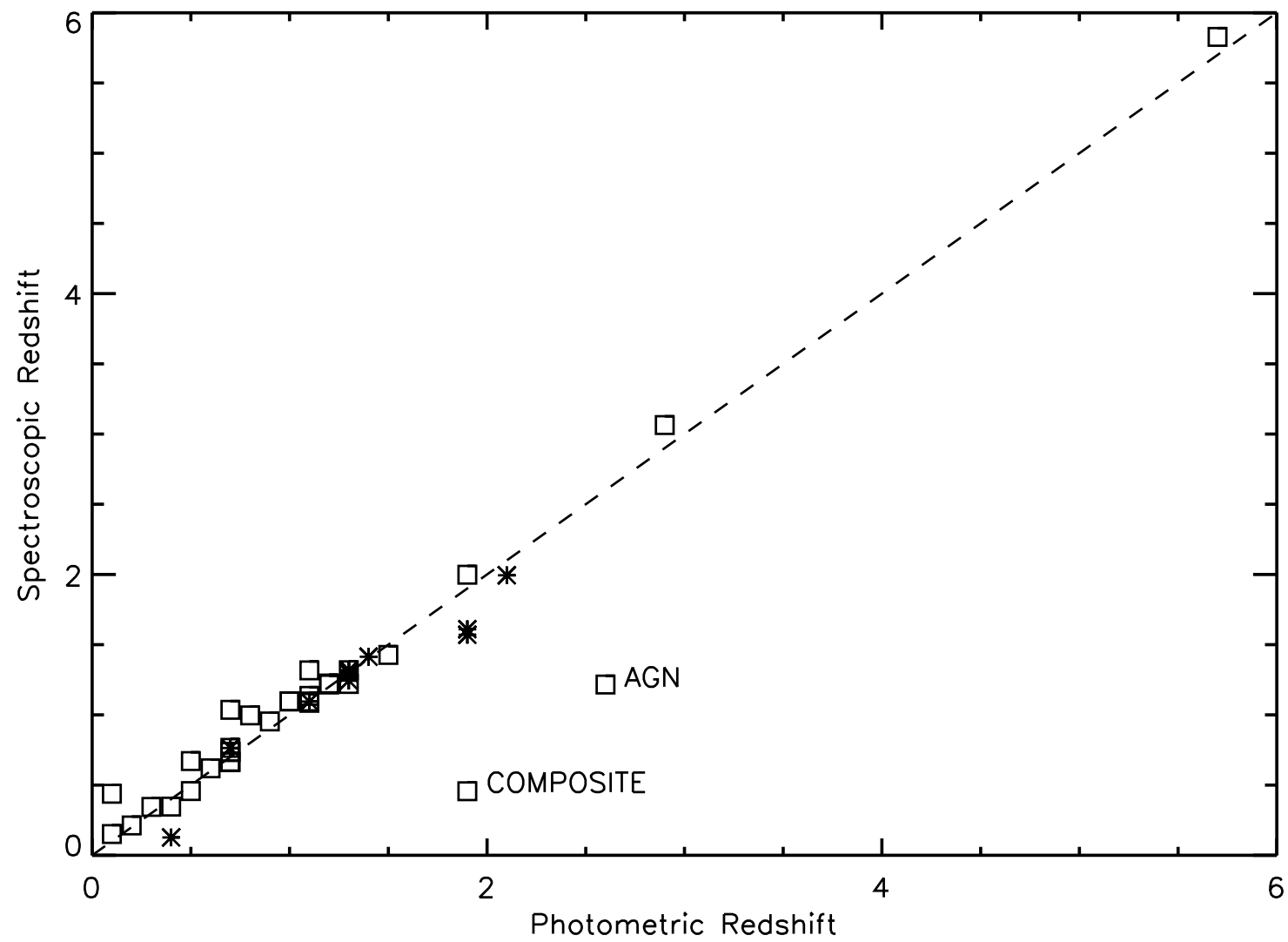

Fig. 6.- A comparison of spectroscopic and photometric redshifts determined in this study. The spectroscopic redshifts come from published an unpublished redshifts from a list utilized in the GOODS program. The square symbols indicate spectroscopic redshifts rated excellent and asterisks redshifts rated good. The dashed line has a slope of 1 and is not a fit to the data. The galaxy marked AGN is an Active Galactic Nucleus and the galaxy marked COMPOSITE is described in the text. Some symbols closely overlie each other 


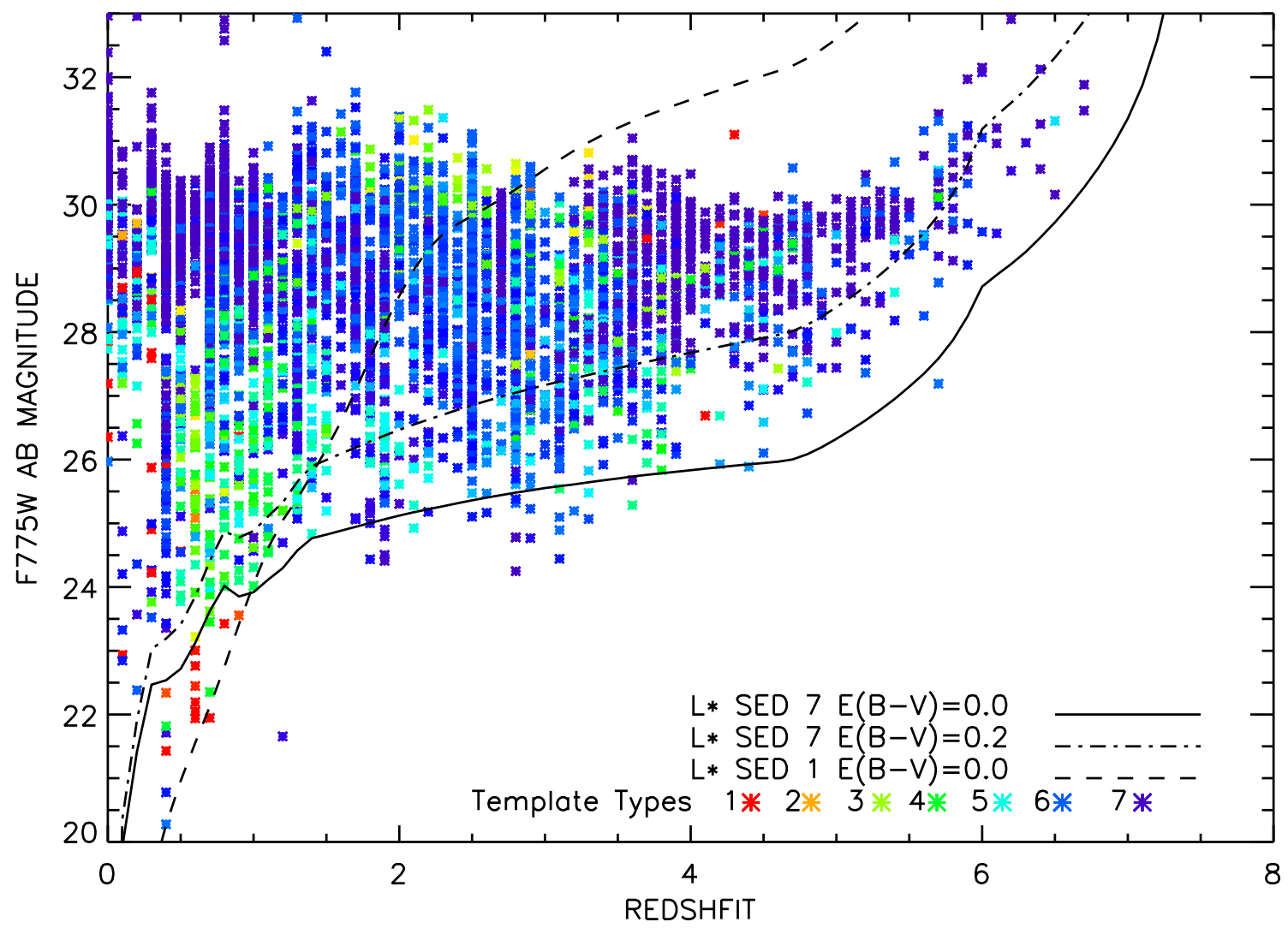

Fig. 7.- Each galaxy in the source extraction is plotted in the F $775 \mathrm{~W}$ versus redshift plane. The symbols are color coded by SED template type as shown in the symbol table at the bottom of the plot. The density of the plot is such that the last galaxy plotted at a particular location determines the color at that location. The F775W AB magnitude tracks for three different $\mathrm{L}^{*}$ galaxies are shown on the plot where $\mathrm{L}^{*}$ is defined as $\mathrm{L}=3.4 \times 10^{10} \mathrm{~L} \odot$. 

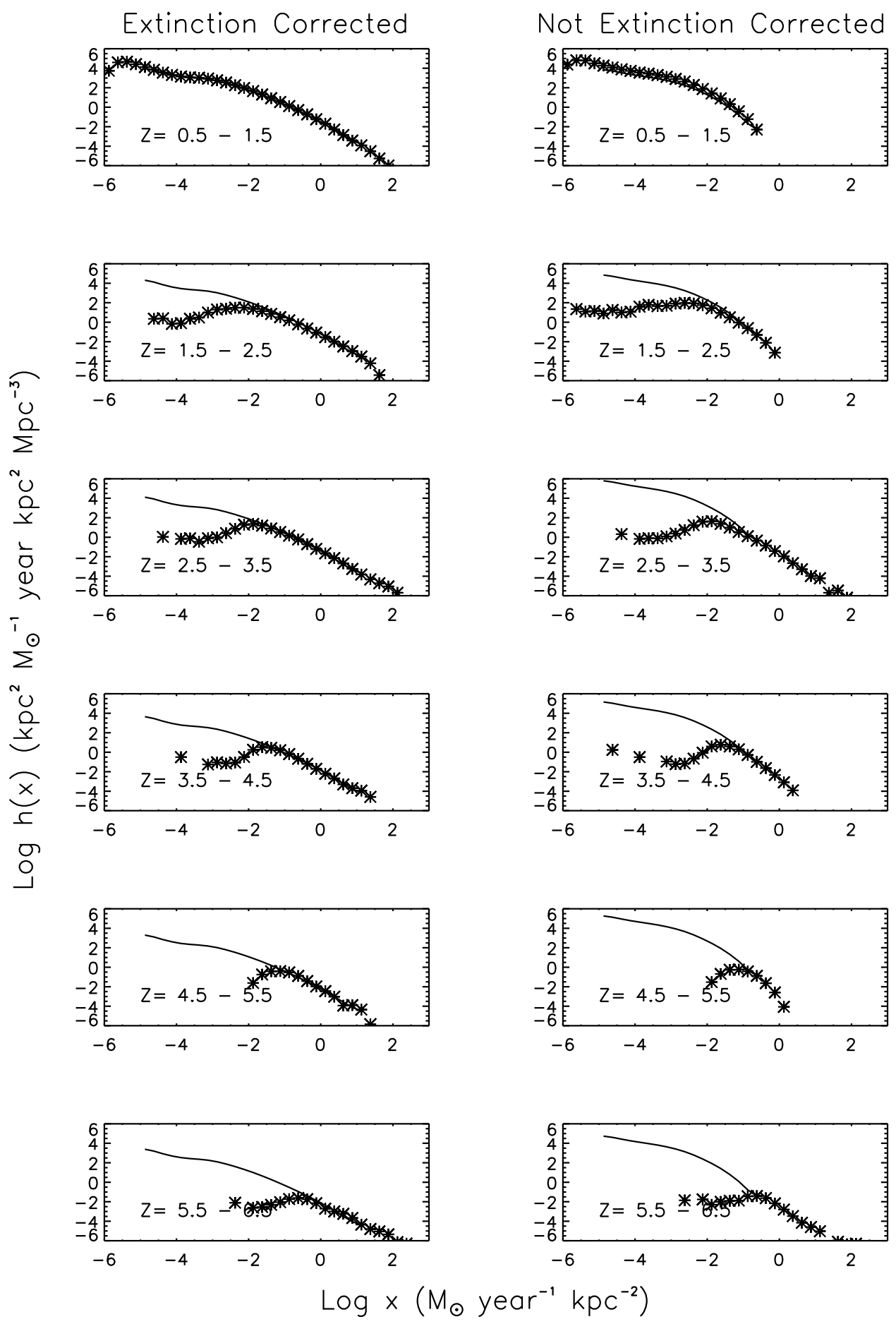

Fig. 8.- Plots of the star formation intensity distribution for each redshift bin. The left hand column intensities have been corrected for extinction while the right hand column intensities have not to show the difference between corrected and non-corrected distributions. 


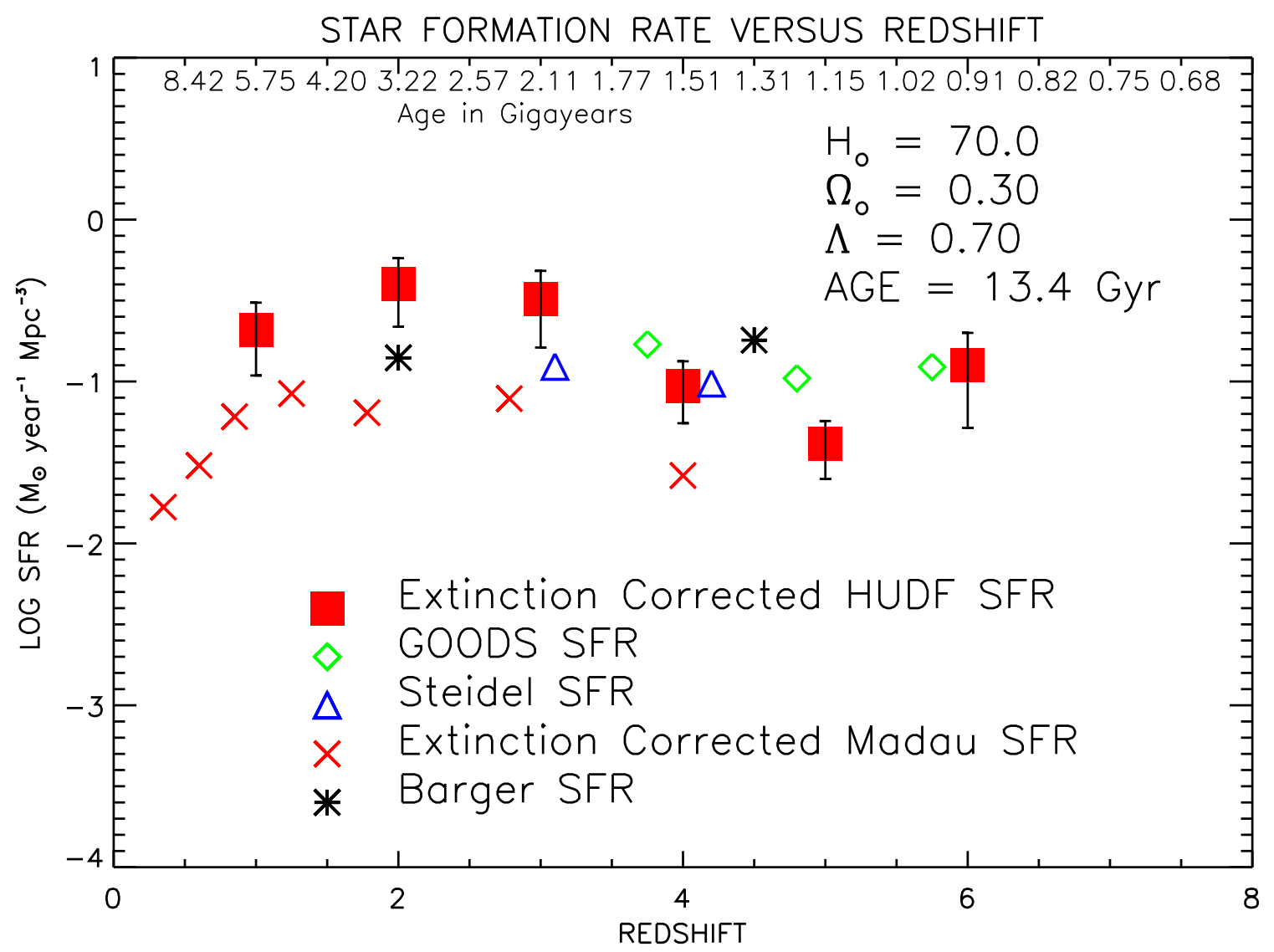

Fig. 9. - The extinction corrected star formation history in the NICMOS region of the HUDF referred to as the NUDF in the text. The SFR history for the much larger GOODS field (Giavalisco et al. 2004) is also shown along with the original Madau data (Madau, Pozzetti, \& Dickinson 1998), the Steidel et al. (1999) z of 3 and 4 ground based results and the Barger, Cowie \& Richards (2000) sub-mm results. The error bars are $1 \sigma$. 


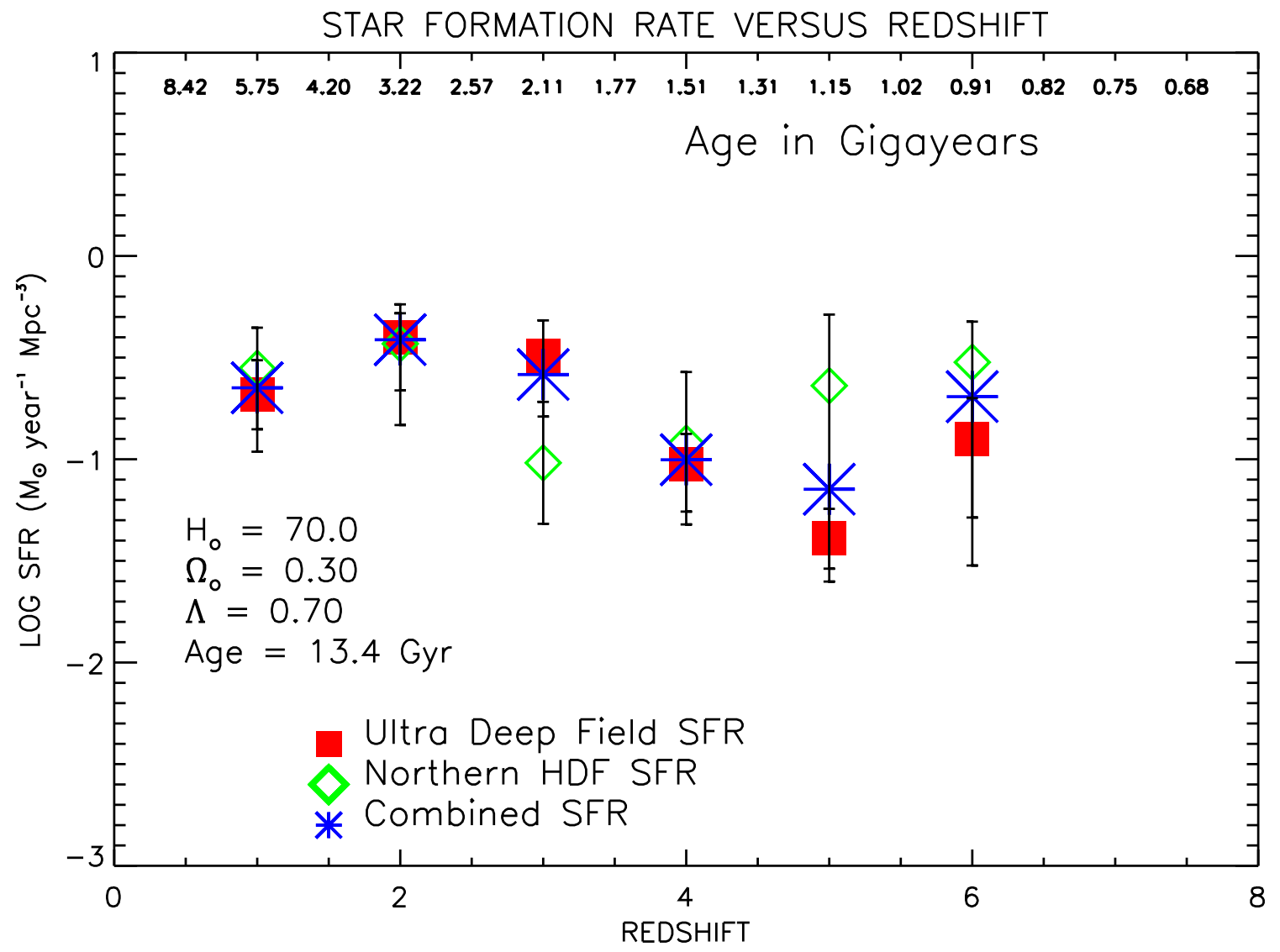

Fig. 10.- The extinction corrected star formation history in the NICMOS region of the HUDF compared to the extinction corrected star formation history in the HDFN. The HDFN SFRs are from Thompson (2003). The error bars are $1 \sigma$. Note that the vertical scale is different from Figure 9. 